\title{
COMPOSIÇÃO DO FITOPLÂNCTON DA BACIA HIDROGRÁFICA DO RIO CAMBORIÚ (SC, BRASIL) DURANTE O VERÃO DE 2005
}

\author{
ANTUNES, A. ${ }^{\text {; }}$ SCHWINGEL, P.R. ${ }^{2}$; BURLIGA, A.L.M. \& URBAN, S. \\ Centro de Ciências Tecnológicas da Terra e do Mar \\ Universidade do Vale do Itajaí - Rua Uruguai, 458. Itajaí, SC, \\ CEP 88302-340, Brasil. \\ 1alineantunes4@yahoo.com; ²schwingel@univali.br
}

\begin{abstract}
Antunes, A.; Schwingel, P.R.; Burliga, A.L.M. \& Urban, S. 2007. Phytoplankton composition of the Camboriú watershed (SC, Brazil) during summer 2005. Braz. J. Aquat. Sci. Technol. 11(2):33-43. ISSN 1808-7035. Pollution of surface freshwaters is one of the main environmental problems that result from antrophic activities. Comprehensive studies concerning water quality, however, demand a general understanding of the related aquatic ecosystems. In particular, water quality can be assessed by analyzing phytoplankton composition and diversity which tend to reflect extreme hydrological pertubations. The present study focus on the qualitative description of microphytoplankton, spatial variability of species composition and the creation of a reference collection of these algae in the Camboriu Watershed (Santa Catarina, Brazil) during summer 2005. Sampling was conducted in 9 stations along the basin. After taxonomic identication of the microphytoplankton, a similarity analysis was applied among all sampling stations. A total of 87 taxa of phytoplankton were identified distributed in classes Cyanophyceae $(n=14)$, Chlorophyceae $(n=19)$, Zygnematophyceae $(n=11)$, Euglenophyceae $(n=8)$, Bacillariophyceae $(n=34)$ and Crysophyceae $(n=1)$. Sampling stations located near the potamon environments showed highest values of total richness and those stations located near the rhithron environments the lowest richness values. The similarity analysis among stations revealed four dissimilar groups, separated into, urban, peri-urban, rural and naturial areas.
\end{abstract}

Keywords: phytoplankton, spacial variation, freshwater, river.

\section{IINTRODUÇÃO}

Os desequilíbrios físico-químicos, biológicos e socioeconômicos ambientais nas Bacias Hidrográficas são um conjunto de fatores relacionados com diferentes tipos de degradação (e.g. lançamento de efluentes sem tratamento, desmatamento em áreas de nascente e matas ciliares, uso inadequado do solo e captação de recursos hídricos sem estudo de vazão), repercutindo na vida aquática. Investigar a estrutura da biota dos rios e áreas adjacentes é fundamental para se conhecer a qualidade ambiental destes ecossistemas e posteriormente estabelecer bases para o entendimento dos processos de planejamento e zoneamento das Bacias Hidrográficas.

Tucci et al. (2004) destacam que a parte taxonômica dos estudos envolvendo o fitoplâncton é de extrema importância, pois a partir da seleção de espécies indicadoras podem ser feitas considerações/relações sobre as condições ambientais. Ao utilizar grupos de espécies como descritoras de determinadas condições ambientais ou, até mesmo, ao elaborar propostas de manejo dos sistemas a partir dos resultados encontrados, são necessários cuidados muito especiais com a identificação e a classificação dos organismos.
A degradação ambiental na Bacia manifesta-se nos recursos hídricos, no solo e na cobertura vegetal, causando alterações na paisagem natural, urbana, periurbana e rural. As interferências ambientais podem causar uma perturbação forte na flora algal (Lobo et al. 2002). No passado, o uso de organismos aquáticos (algas e invertebrados) para monitorar rios foi de importância secundária comparado a variáveis físicas e químicas, entretanto, existe uma tendência ao uso de algas em estudos de monitoramento mais recentes (Lobo et al. 2002; Miranda et al. 2003).

As análises da estrutura das populações de algas vêm sendo utilizadas para monitorar qualidade de água de rio em diversos países (Alemanha, Bélgica, França e Inglaterra). A avaliação da qualidade da água pode ser feita através da composição e diversidade de espécies de fitoplâncton, as quais são indicadoras de perturbações hidrológicas extremas, visto que a quantidade e qualidade de água produzem alterações rápidas na estrutura das populações (Hürlimann et al., 1991).

A Bacia Hidrográfica do Rio Camboriú drena uma área de $200 \mathrm{~km}^{2}$ e fica localizada nos municípios de Camboriú, onde se encontra mais de $90 \%$ das nascentes, e Balneário Camboriú (SC). O rio possui cerca de $40 \mathrm{~km}$ de extensão e deságua no extremo sul da praia 
de Balneário Camboriú, com uma largura de, aproximadamente 120 metros (Pereira-Filho et al., 2001). Por se tratar do único manancial, a Bacia do Rio Camboriú é importante para essas cidades, as quais dependem do aporte de água do rio e seus tributários para o desenvolvimento agropecuário, sócio-econômico e abastecimento da população. Esta bacia hidrográfica vem sofrendo com a degradação ambiental provocada pela expansão na área cultivada, destacando-se a rizicultura, exploração de rocha, madeira para as carvoarias, além da pastagem para o gado (Urban, 2003). Assim, é necessário se conhecer os níveis de qualidade ambiental nas diferentes regiões da Bacia Hidrográfica do Rio Camboriú, os quais poderão subsidiar programas de gestão ambiental neste ecossistema límnico. A estrutura de assembléias de algas límnicas na Bacia Hidrográfica do Rio Camboriú é desconhecida, não existindo registros de estudos sobre fitoplâncton neste ambiente. Portanto, o presente estudo teve como objetivo avaliar a composição taxonômica das assembléias fitoplanctônicas encontradas no rio Camboriú, contribuindo-se assim para o conhecimento da diversidade destes organismos e de sua variação espacial durante o verão.

\section{METODOLOGIA}

O presente trabalho foi desenvolvido na Bacia Hidrográfica do Rio Camboriú (SC), onde foram selecionados 9 pontos amostrais previamente georeferenciados (Tabela 1). A Figura 1 mostra a distribuição dos pontos amostrais, compreendendo os pontos de 2 a 10 estudados por Urban (2003), o qual analisou a qualidade da água da Bacia Hidrográfica do Rio
Camboriú, SC. O ponto amostral 1 não foi incluído neste trabalho por se localizar em área estuarina, o que não é alvo do presente estudo. A localização dos pontos amostrais procurou abranger de forma mais ampla possível a bacia hidrográfica, compreendendo áreas urbanas, peri-urbanas, rurais e naturais. A coleta foi realizada em fevereiro de 2005 , compreendendo a estação de verão, na qual o fitoplâncton se encontra mais abundante.

Para a análise qualitativa, as amostras foram coletadas com rede de plâncton com abertura de maIha de 25 micrômetros, compreendendo assim o microfitoplâncton ( 20 a 200 micrômetros). O volume filtrado foi de aproximadamente 50 litros por ponto amostral. As amostras foram coletadas na região central da secção transversal do rio, abrangendo a camada sub-superficial. As amostras foram acondicionadas em frascos de $500 \mathrm{ml}$ e fixadas com formol $4 \%$.

A identificação taxonômica das espécies foi realizada em microscópio binocular Olympus, em aumentos de até 1000 vezes, até o menor taxon possível. A análise quali-quantitativa das amostras envolveu a confecção de lâminas e posterior identificação até a suficiência amostral, ou seja, até o esgotamento da identificação de novos taxa no ponto amostral analisado, fato que ocorreu após o exame de aproximadamente 6 lâminas por ponto. A identificação dos taxa foram baseadas nos seguintes autores, entre outros: Germain (1981), Bourrelly (1985), Rosa \& Miranda-Kiesslich (1988), Hegewald (2000), Krammer (2000), LangeBertalot (2001), Lobo et al. (2002), Bicudo \& Menezes (2006) e Sant'Anna et al. (2006), seguindo a classificação taxonômica de Hoek et al. (1995). As imagens dos taxa identificados foram realizadas com máquina fotográfica Nikon acoplada em microscópio óptico Olympus, utilizando Analisador de Imagens Image - Pro Plus ${ }^{\circ}$.

Tabela 1 - Posições geográficas dos pontos de coleta de fitoplâncton na Bacia Hidrográfica do Rio Camboriú (SC).

\begin{tabular}{ccc|c|c}
\hline \hline \multirow{2}{*}{$\begin{array}{c}\text { Ponto } \\
\text { Amostral }\end{array}$} & \multirow{2}{*}{ Nome do Ponto } & Característica & \multicolumn{2}{|c}{ Posição Geográfica } \\
\cline { 4 - 5 } & & & Latitude & Longitude \\
\hline 2 & Rio Peroba & Urbano & $27^{\circ} 00^{\prime} 34,4^{\prime \prime}$ & $48^{\circ} 38^{\prime} 47,5^{\prime \prime}$ \\
3 & Adutora CASAN & Urbano & $27^{\circ} 01^{\prime} 14,1^{\prime \prime}$ & $48^{\circ} 39^{\prime} 44,3^{\prime \prime}$ \\
4 & Rio Pequeno & Peri-urbano & $27^{\circ} 01^{\prime} 26,3^{\prime \prime}$ & $48^{\circ} 38^{\prime} 29,4^{\prime \prime}$ \\
5 & Início Rio Camboriú & Rural & $27^{\circ} 05^{\prime} 29,6^{\prime \prime}$ & $48^{\circ} 41^{\prime} 34,1^{\prime \prime}$ \\
6 & Macacos & Natural & $27^{\circ} 07^{\prime} 14,9^{\prime \prime}$ & $48^{\circ} 41^{\prime} 59,5^{\prime \prime}$ \\
7 & Caetês & Natural & $27^{\circ} 07^{\prime} 21,9^{\prime \prime}$ & $48^{\circ} 43^{\prime} 08,8^{\prime \prime}$ \\
8 & Louro & Natural & $27^{\circ} 06^{\prime} 59,7^{\prime \prime}$ & $48^{\circ} 44^{\prime} 55,6^{\prime \prime}$ \\
9 & Limeira & Natural & $27^{\circ} 06^{\prime} 22,1^{\prime \prime}$ & $48^{\circ} 46^{\prime} 24,0^{\prime \prime}$ \\
10 & Bloqueio 17 Petrobrás & Rural & $27^{\circ} 03^{\prime} 25,4^{\prime \prime}$ & $48^{\circ} 42^{\prime} 14,3^{\prime \prime}$ \\
\hline \hline
\end{tabular}




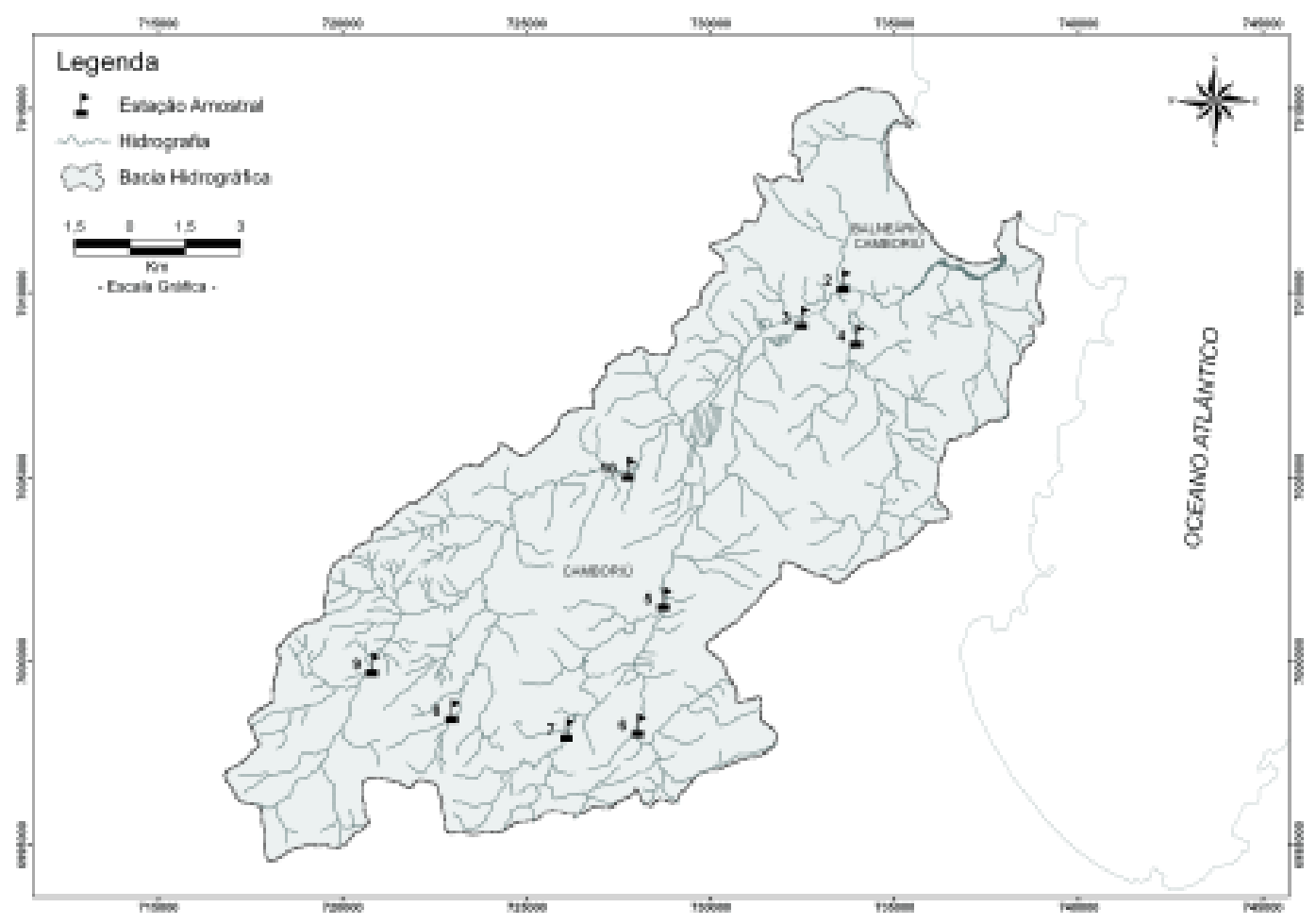

Figura 1 - Localização dos pontos amostrais na Bacia Hidrográfica do Rio Camboriú (SC).

A riqueza específica do fitoplâncton foi analisada de duas formas: riqueza específica total (somatório das espécies) para cada ponto amostral e para cada classe fitoplanctônica.

Com o intuito de detectar similaridades entre os pontos amostrais foi realizada uma Análise de Cluster do programa Statistica. Esta análise foi baseada na matriz de presença e ausência dos taxa de microfitoplâncton identificados nos 9 pontos amostrais analisados. A medida de distância e o método de ligação utilizados para a análise de similaridade foram a Manhattan e o Ward, respectivamente.

\section{RESULTADOS E DISCUSSÃO}

\section{Composição do Fitoplâncton}

Foram identificados 80 taxa do fitoplâncton na Bacia Hidrográfica do Rio Camboriú distribuídos nos 9 pontos amostrais analisados (Tabela 2). A maior amplitude na distribuição na área estudada foi apresentada por Surirella sp., ocorrendo em oito pontos amostrais, seguido de Ulnaria ulna, que foi encontrada em seis pontos. Lyngbya sp., Oscillatoria sp3, Desmodesmus opoliensis, Euglena acus, Amphipleura lindheimerii, Melosira varians, Navicula sp. e Pinnularia sp. também foram freqüentes no ambiente. Dos demais taxa identificados, $59 \%$ ocorreram em apenas um ponto amostral. Os taxa identificados foram distribuídos em seis classes, a saber: Cyanophyceae $(n=11)$, Chlorophyceae $(n=16)$, Zygnematophyceae $(n=11)$, Euglenophyceae $(n=8)$, Bacillariophyceae $(n=33)$ e Crysophyceae $(n=1)$. Abaixo é apresentada uma lista com a classificação taxonômica, bem como suas respectivas variações morfométricas.

\section{Divisão Cyanophyta \\ Classe Cyanophyceae \\ Coelosphaerium sp. \\ Lyngbyasp.}

Merismopedia convoluta Brébisson (Fig. 2)

Diâmetro celular 4,1 - 5,1 mm

Merismopedia glauca (Ehrenberg) Nägeli (Fig. 3)

Diâmetro celular 2,8 - 3,4 mm

Oscillatoria sp1

Oscillatoria sp2

Oscillatoria sp3

Phormidium sp.

Planktolyngbya sp.

Pseudanabaena sp.

Scytonema sp. 


\section{Divisão Chlorophyta}

Classe Chlorophyceae

Coelastrum polychordum (Kosikov) Hindák

Célula externa: diâmetro $10 \mathrm{~mm}$; colônia: diâmetro $43 \mathrm{~mm}$

Desmodesmus bicaudatus Hegewald (Fig. 4)

Comprimento da colônia $13 \mathrm{~mm}$; largura celular $12,8 \mathrm{~mm}$

Desmodesmus gutwinskii Chod. (Fig. 5)

Comprimento da colônia 16,1 - 16,3 mm; largura celular 7,8 - $8 \mathrm{~mm}$

Desmodesmus heteracanthus Hegewald (Fig. 6)

Comprimento da colônia 62,7 mm;

largura celular 13,4 mm

Desmodesmus opoliensis (P. Richter) Hegewald

(Fig. 7)

Comprimento da colônia 14,2 - 20,9 mm; largura celular 14,7-20,3 mm

Desmodesmus protuberans (Fritsch \& Rich) E. Hegewald (Fig. 8)

Comprimento da colônia 16,4 - 39 mm; largura celular 7,8 - 12,7 mm

Eudorina sp.

Monoraphidium sp1

Monoraphidium sp2

Oedogonium sp.

Oocystis sp.

Pediastrum duplex var. duplex Meyen (Fig. 9)

Célula interna: comprimento $26,7 \mathrm{~mm}$; lar gura $31,5 \mathrm{~mm}$. Célula externa:

comprimento $38,2 \mathrm{~mm}$; largura $27,2 \mathrm{~mm}$

Pediastrum simplex Meyen (Fig. 10)

Comprimento celular $22 \mathrm{~mm}$; largura

celular 7,5 mm

Diâmetro total do cenóbio $63,8 \mathrm{~mm}$

Pediastrum tetras (Ehrenberg) Ralfs (Fig. 11)

Célula interna: comprimento $6,5 \mathrm{~mm}$;

largura 6,8 mm

Célula externa: comprimento $10,3 \mathrm{~mm}$;

largura $10,1 \mathrm{~mm}$

Stigeoclonium sp.

Volvulina sp.

Classe Zygnematophyceae

Closterium sp1

Closterium sp2

Cosmarium sp1

Cosmarium sp2

Cosmarium sp3

Euastrum sp.

Gonatozygon sp.

Hyalotheca dissiliens Brébisson ex Ralfs (Fig.12)

Célula: comprimento $3,9 \mathrm{~mm}$; largura 12,2 $\mathrm{mm}$

Mougeotia sp.

Pleurotaenium sp.
Spirogyra sp.

Divisão Euglenophyta

Classe Euglenophyceae

Euglena acus Ehrenberg (Fig. 13)

Comprimento celular $80,1-155 \mathrm{~mm}$; largura 10,2 - $20 \mathrm{~mm}$;

Euglena sp.

Lepocinclis sp.

Phacus longicauda (Ehrenberg) Dujardin (Fig. 14)

Comprimento celular $120 \mathrm{~mm}$; largura celular $45 \mathrm{~mm}$;

Phacus sp.

Phacus tortus (Lemmermann) Skvortsov (Fig. 15)

Comprimento celular $109 \mathrm{~mm}$; largura

celular $38,8 \mathrm{~mm}$;

Trachellomonas armata (Ehrenberg) Stein

(Fig.16)

Comprimento celular: 28 - 47,9 mm; largura celular 22 - 33,8 mm;

Trachellomonas sp.

\section{Divisão Heterokontophyta}

Classe Bacillariophyceae

Achnanthes inflata Kützing (Fig. 17)

Comprimento celular 59,1 - 76,5 mm; largura 19 - 29,5 mm; 9 estrias em $10 \mathrm{~mm}$

Amphipleura lindheimerii Grunow (Fig. 18) Comprimento celular 127,9 - $185 \mathrm{~mm}$; largura 19,3 - 21,6 mm

Aulacoseira granulata (Ehrenberg) Simonsen

(Fig. 19)

comprimento celular 22,6 - 26,7 mm; largura 4 - 7,7 mm

Cymbella tumida (Brébisson) Van Heurck (Fig.

20)

Comprimento celular 62,5 - $85 \mathrm{~mm}$; largura 19,6 - $20 \mathrm{~mm}$; 9 estrias em $10 \mathrm{~mm}$;

Encyonemasp.

Eunotia sp.

Fragilaria capucina Desmaziéres (Fig. 21)

Comprimento celular $32,5 \mathrm{~mm}$; largura 4,2 $\mathrm{mm} ; 13$ estrias em $10 \mathrm{~mm}$

Fragilaria goulardii Brébisson (Fig. 22) Comprimento celular 106,8 - 113,6 mm; largura 6 - 9,3 mm; 11 - 12 estrias em 10 $\mathrm{mm}$

Gomphonema gracile Ehrenberg (Fig. 23) Comprimento celular 39,1 mm; largura 9,9 $\mathrm{mm}$; 11 estrias em $10 \mathrm{~mm}$

Gomphonema parvulum Kützing (Fig. 24)

Comprimento celular $23,9 \mathrm{~mm}$; largura 7 $\mathrm{mm}$; 12 estrias em $10 \mathrm{~mm}$

Gomphonema sp.

Gyrosigmasp.

Hydrosera whampoensis (Schw.) Deby (Fig. 25) 
Comprimento do eixo apical 55 - $59 \mathrm{~mm}$

Melosira ambigua O. Muller (Fig. 26)

Célula: comprimento 18,7 - $24 \mathrm{~mm}$;

largura 6,8- $7 \mathrm{~mm}$

Melosira sp.

Melosira varians C. A. Agardh (Fig. 27)

Célula: comprimento $26,2-33,7 \mathrm{~mm}$;

largura $17-23,7 \mathrm{~mm}$

Navicula sp.

Neidium sp.

Nitzschia clausii Hantzsch (Fig. 28)

Comprimento celular $69,5 \mathrm{~mm}$; largura 4,4 $\mathrm{mm}$

Nitzschia fasciculata Grunow

Comprimento $85 \mathrm{~mm}$; largura $6 \mathrm{~mm} ; 5$

fíbulas em $10 \mathrm{~mm}$

Nitzschia palea (Kütz.) W. Smith

Comprimento $53 \mathrm{~mm}$; largura $6 \mathrm{~mm} ; 14$

estrias em $10 \mathrm{~mm}$

Nitzschia sp.

Comprimento 62 - $159 \mathrm{~mm}$; largura 11,8 -

24,3 mm; 8 - 10 estrias em 10 mm

Pinnularia biceps Gregory

Comprimento celular $71 \mathrm{~mm}$; largura 14

$\mathrm{mm} ; 12$ estrias em $10 \mathrm{~mm}$

Pinnularia cf maior (Kütz.) Cleve (Fig. 29)

Comprimento celular 245 - $274 \mathrm{~mm}$;

largura 37 - 38,9 mm; 6 - 7 estrias em 10

$\mathrm{mm}$

Pinnularia mesolepta (Enrenberg) W. Smith

Comprimento celular $70 \mathrm{~mm}$; largura 9

$\mathrm{mm} ; 12$ estrias em $10 \mathrm{~mm}$

Pinnularia sp.

Placoneis disparilis (Hustedt) Metzeltin e Lange-

Bertalot (Fig. 30)

Comprimento celular $37,8 \mathrm{~mm}$; largura

$14,9 \mathrm{~mm}$; 13 estrias centrais em $10 \mathrm{~mm}$;

Pleurosira laevis (Ehrenberg) Compère (Fig. 31)

Diâmetro celular $77 \mathrm{~mm}$

Surirella sp.

Synedra sp.

Terpsinoe musica Ehrenberg (Fig. 32)

Comprimento celular $119,7 \mathrm{~mm}$; largura 45 $\mathrm{mm}$

Tryblionella sp.

Ulnaria ulna (Nitzsch) Ehr. (Fig. 33)

Comprimento celular 125 - 399,2 mm; largura 6,8 - $14 \mathrm{~mm} ; 8$ - 11 estrias em 10 $\mathrm{mm}$

\section{Classe Crysophyceae}

Synura sp.
Variação Espacial da Composição do Fitoplâncton

A riqueza específica total da seis classes identificadas (Cyanophyceae, Chlorophyceae, Zygnematophyceae, Euglenophyceae, Bacillariophyceae e Chrysophyceae), podem ser visualizadas na Figura 34. Segundo Reviers (2006), as diatomáceas (Bacillariophyceae) estão amplamente distribuídas na natureza, uma vez que, assim como as cianobactérias e as algas verdes, estão aptas a colonizar a maioria dos ambientes aquáticos. Depois das bactérias, são provavelmente os organismos aquáticos de distribuição mais ampla, exercendo um papel importante como produtores primários. As algas verdes (Chlorophyceae e Zygnematophyceae) e as cianobactérias (Cyanophyceae) obtiveram valores de riqueza total entre 15 e 25 espécies. O grupo Euglenophyceae, que ocorre especialmente em meios ricos em matéria orgânica, obteve valor de riqueza total igual a 16. Chrysophyceae é a classe menos representativa, tendo sido encontrado apenas um taxa. Os pontos amostrais com maior riqueza foram ponto $2(n=$ 19) e o ponto $4(n=30)$, localizados em áreas urbana e peri-urbana respectivamente, sendo que o ponto $8(n=7)$ obteve a menor riqueza, localizado em uma área natural (Figura 35).

Na região potamal do Rio Camboriú, trecho mais próximo à foz, a velocidade das águas do rio é baixa, em razão do menor declive, favorecendo a concentração de nutrientes, e conseqüentemente o crescimento do fitoplâncton. Os nutrientes representam o alimento para o fitoplâncton, e as suas altas concentrações auxiliam no crescimento. Os pontos amostrais 2, 3, 4, 5 e 10 estão inseridos nesta região e de um modo geral apresentam valores de riqueza elevados. Os pontos amostrais 6, 7, 8 e 9 estão localizados na região ritral, onde teoricamente a velocidade das águas é maior em função do declive, impedindo a concentração de nutrientes e também o estabelecimento das assembléias fitoplanctônicas. Nestes pontos, em geral, foram constatados os menores valores de riqueza específica. $O$ ponto amostral 4 , apesar de ter sido constatada uma maior riqueza, é um local considerado crítico, pois possui pouca mata ciliar e elevado grau de poluição, e conseqüentemente altas concentrações de nutrientes provenientes dos esgotos domésticos lançados no rio. $O$ ponto amostral 8 , no qual se encontra a menor riqueza total, está inserido em uma região altamente preservada, que favoreceria o crescimento do fitoplâncton. Entretanto, o mesmo se localiza em uma região considerada ritral (123 metros de altitude), onde a concentração de nutrientes deve ser menor (ambiente oligotrófico) e possui também maior velocidade da corrente. A partir disso, pode-se inferir que as assembléias fitoplanctônicas não conseguem se estabelecer nes- 
Tabela 2 - Matriz de presença e ausência dos taxa de fitoplâncton identificados na Bacia Hidrográfica do Rio Camboriú para 9 pontos amostrais, no verão de 2005 (presença= 1; ausência= 0).

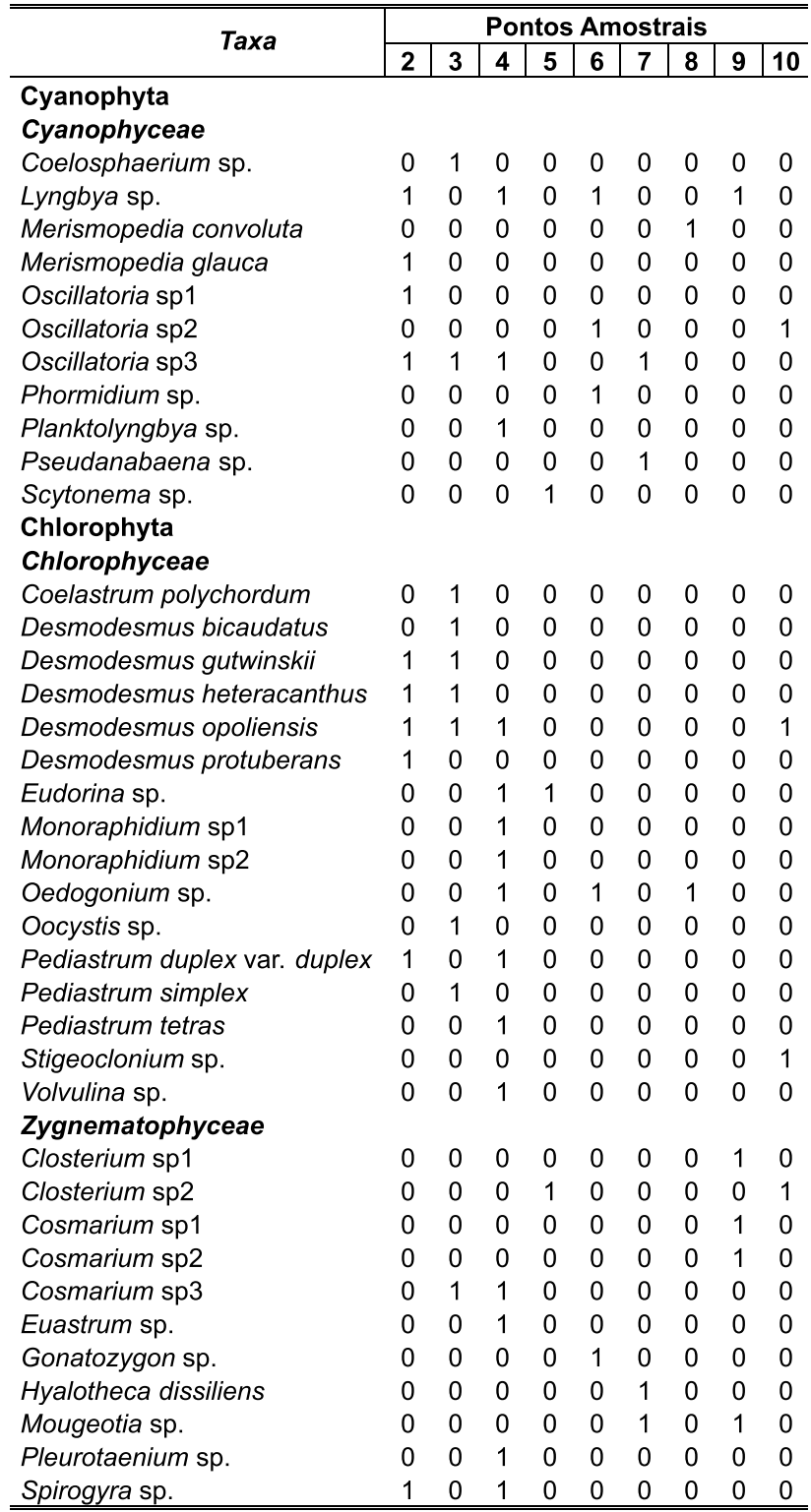

tas áreas, fato corroborado pela baixa riqueza encontrada.

A riqueza específica do fitoplâncton nas 9 estações amostrais, para as diferentes classes identificadas, pode ser visualizada na Figura 36 . O ponto amostral 2 apresenta a maior riqueza específica para a classe Cyanophyceae, sendo que a mesma decresce levemente em direção à região montante (Fig. 36-a). Para Chlorophyceae, os pontos amostrais 2, 3 e 4 (região jusante) apresentam valores destacadamente maiores de riqueza (Fig. 36-b). Este fato sugere que a ocorrência desta classe está relacionada a região com maior atividade antrópica, ou seja, áreas urbanas e periurbanas. A riqueza para a classe Zygnematophyceae não apresenta padrão definido como mostra a Figura

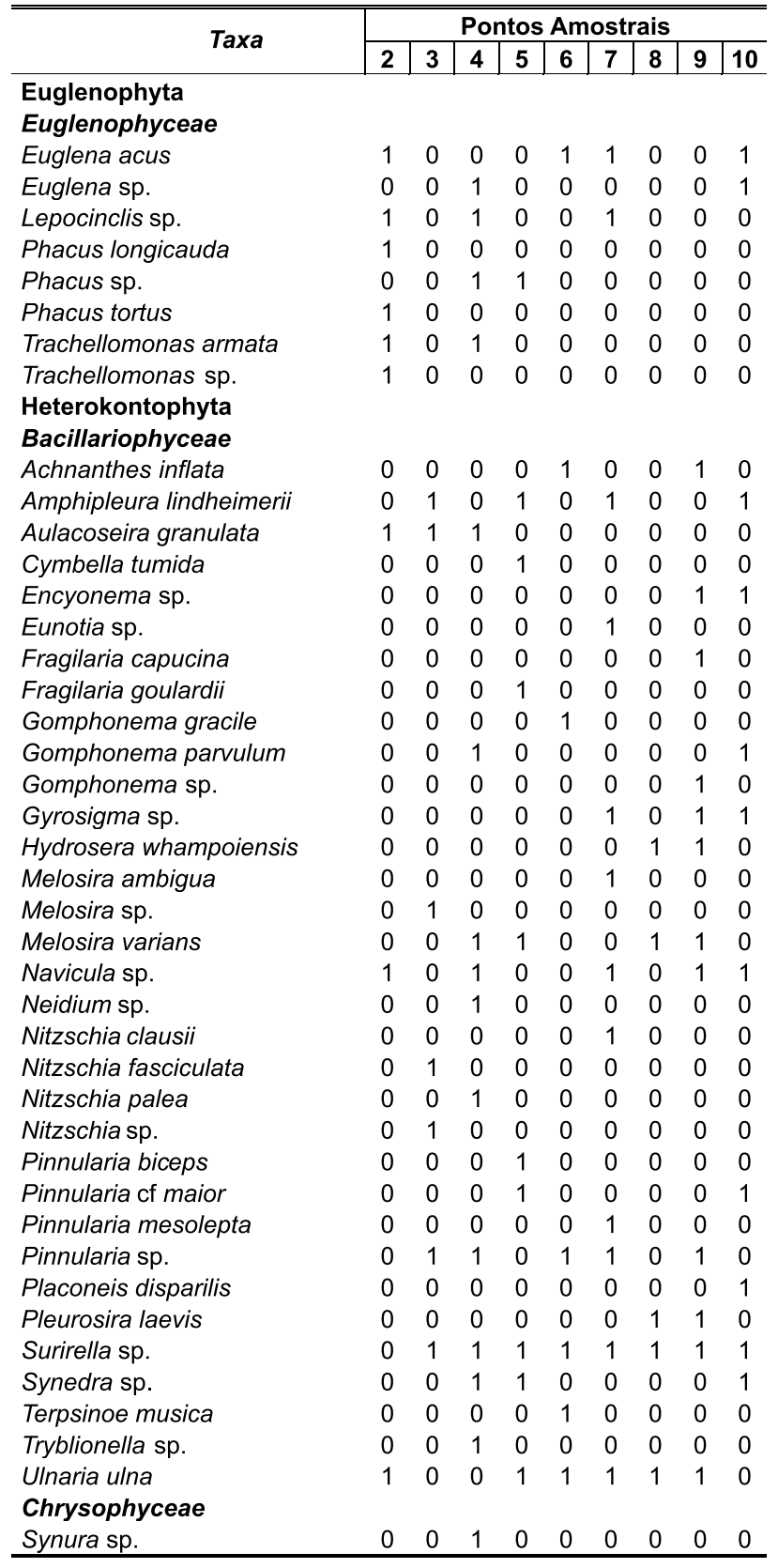

36-c. A classe Euglenophyceae foi melhor representada nos pontos 2 e 4 (Fig. 36-d), locais afetados por poluição decorrente de esgotos domésticos, confirmando o fato desta classe compreender organismos típicos de águas ricas em matéria orgânica. Bacillariophyceae é a mais representativa das seis classes, exibindo valores de riqueza elevados em relação às outras classes, situação que ocorre em quase todos os pontos amostrais (Fig. 36-e). A classe Chrysophyceae, representada na Figura 36-f, teve apenas uma ocorrência no ponto amostral 4 (Synura sp.). 

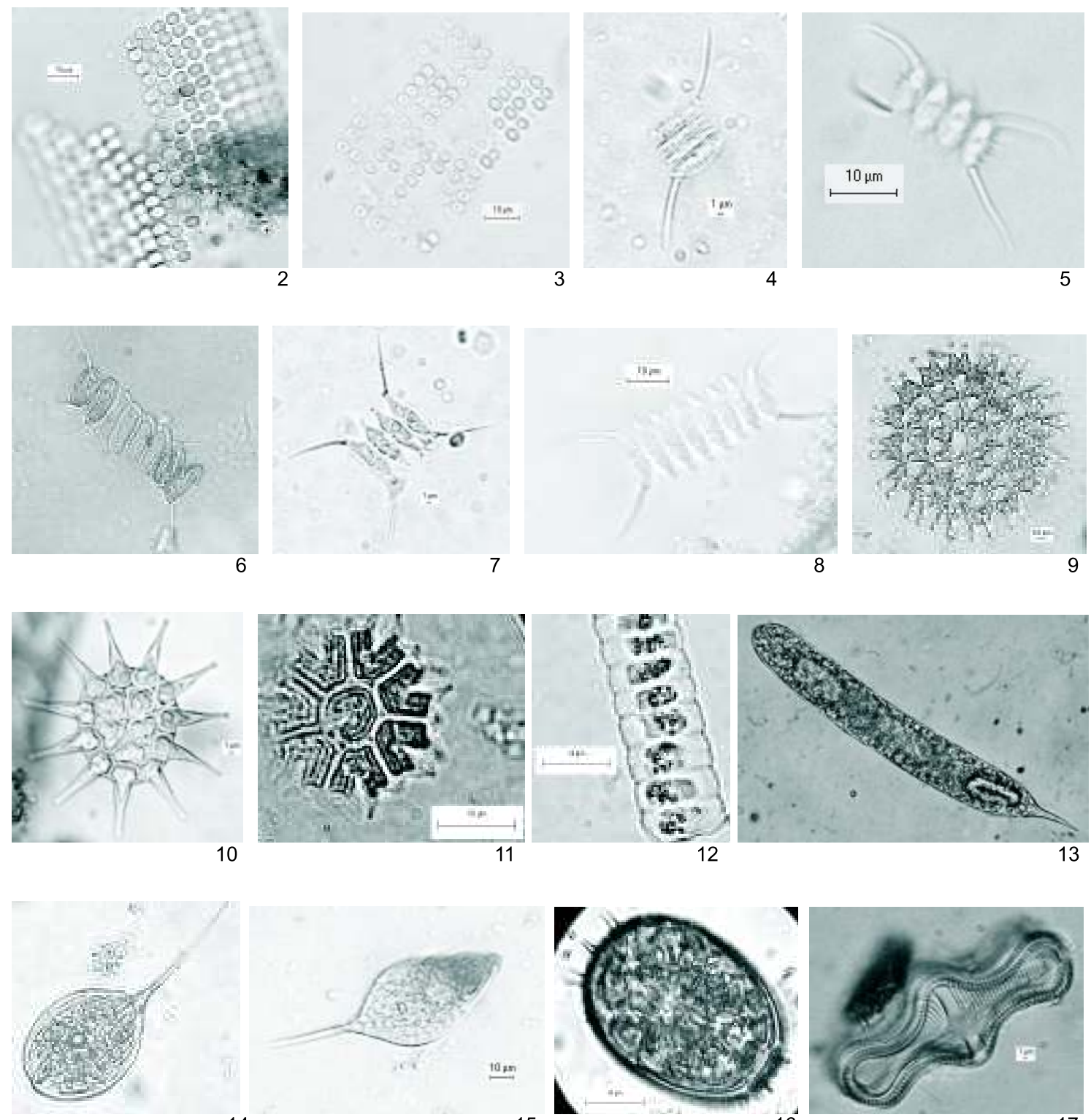

14
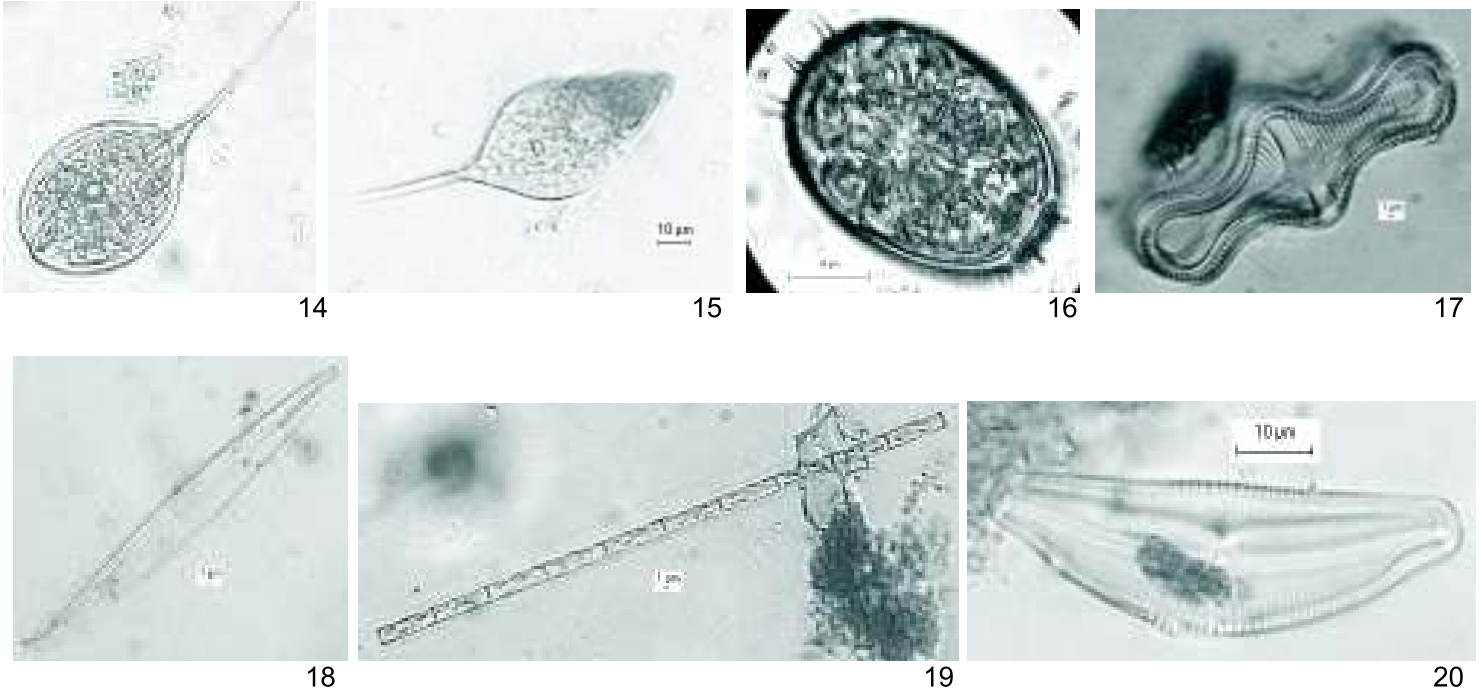

19

20

Figuras 2-20 - (2) Merismopedia convoluta; (3) Merismopedia glauca; (4) Desmodesmus bicaudatus; (5) Desmodesmus gutwinskii; (6) Desmodesmus heteracanthus; (7) Desmodesmus opoliensis; (8) Desmodesmus protuberans; (9) Pediastrum duplex var. duplex; (10) Pediastrum simplex; (11) Pediastrum tetras; (12) Hyalotheca dissiliens; (13) Euglena acus; (14) Phacus longicauda; (15) Phacus tortus; (16) Trachellomonas armata; (17) Achnanthes inflata; (18) Amphipleura lindheimerii; (19) Aulacoseira granulata; (20) Cymbella tumida. $($ Escala $=10$ um $)$. 

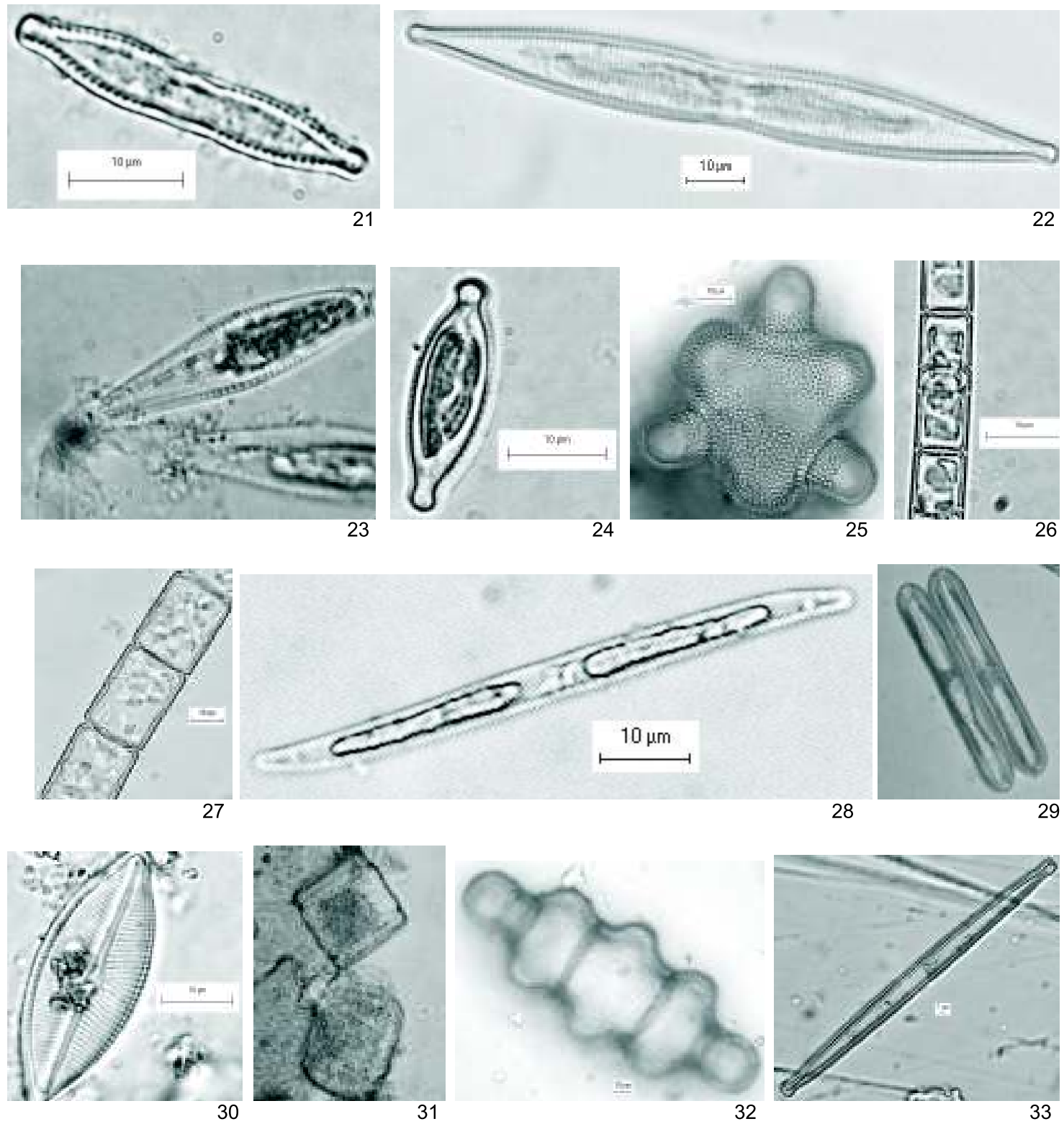

Figuras 21-33 - (21) Fragilaria capucina; (22) Fragilaria goulardii; (23) Gomphonema gracile; (24) Gomphonema parvulum; (25) Hydrosera whampoiensis; (26) Melosira ambigua; (27) Melosira varians; (28) Nitzschia clausii; (29) Pinnularia cf maior; (30) Placoneis disparilis; (31) Pleurosira laevis; (32) Terpsinoe musica; (33) Ulnaria ulna. (Escala = $10 \mathrm{~mm}$ ).

Apenas 6 espécies $(25 \%)$ de diatomáceas encontradas neste estudo foram também identificadas no trabalho de Burliga et al. (2005), em estudo sobre diatomáceas epilíticas no rio Itajaí-Mirim, localizado próximo à Bacia do Rio Camboriú, são elas: Melosira varians, Gomphonema parvulum, Hydrosera whampoiensis, Pinnularia mesolepta, Placoneis disparilis e Pleurosira laevis. A espécie Euglena acus, encontrada na área de rizicultura (ponto amostral 10), foi a única espécie também identificada no trabalho de Tamanaha (2004), que trata da estrutura das assembléias fitoplanctônicas e testes de inibição de crescimento algal, avaliando os efeitos dos herbicidas e inse- ticidas utilizados na cultura do arroz irrigado na região de Itajaí. Esses estudos mostram que apesar da proximidade geográfica, a composição específica fitoplanctônica dos rios de uma região pode variar consideravelmente.

Para verificar a similaridade entre os pontos amostrais foi utilizada uma Análise de Cluster (agrupamento), baseada na matriz de binária (presença e ausência) dos 80 taxa de fitoplâncton identificados no Rio Camboriú (Figura 37). O resultado revelou a existência de quatro grupos. O primeiro grupo envolve os pontos amostrais 6,7 , 8, e 9, os quais estão localizados em áreas naturais, compreendendo as áreas mais preser- 


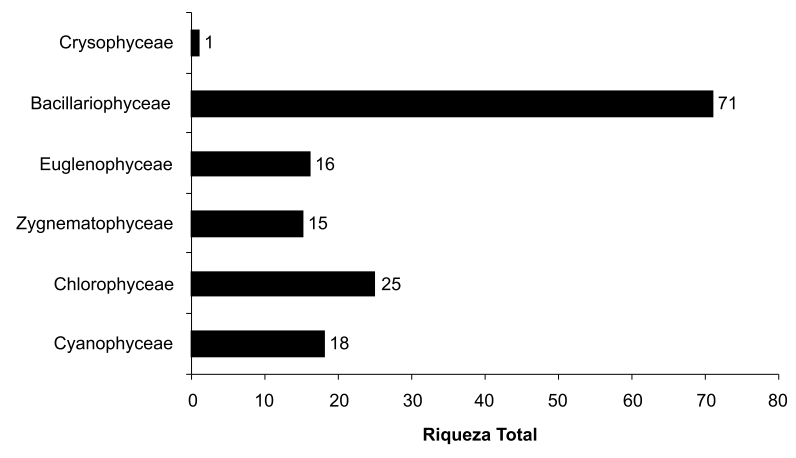

Figura 34 - Riqueza total (soma das riquezas observadas para os 9 pontos amostrais) para diferentes classes do fitoplâncton encontradas na Bacia Hidrográfica do Rio Camboriú (SC) para o verão de 2005

\section{Cyanophyceae}

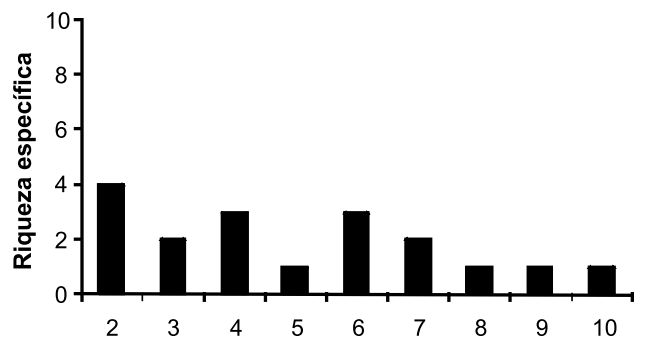

Chlorophyceae

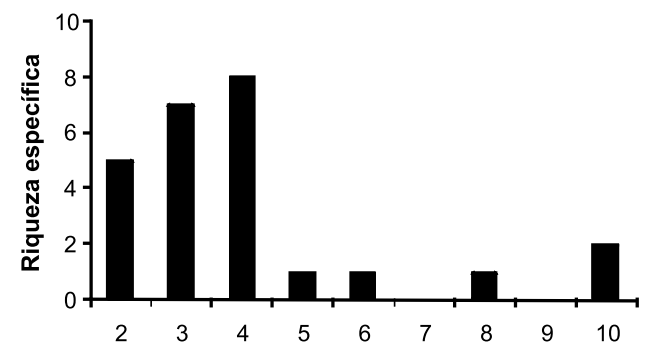

Zygnematophyceae

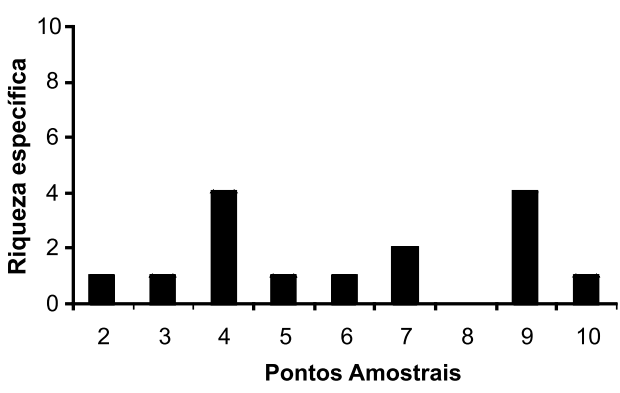

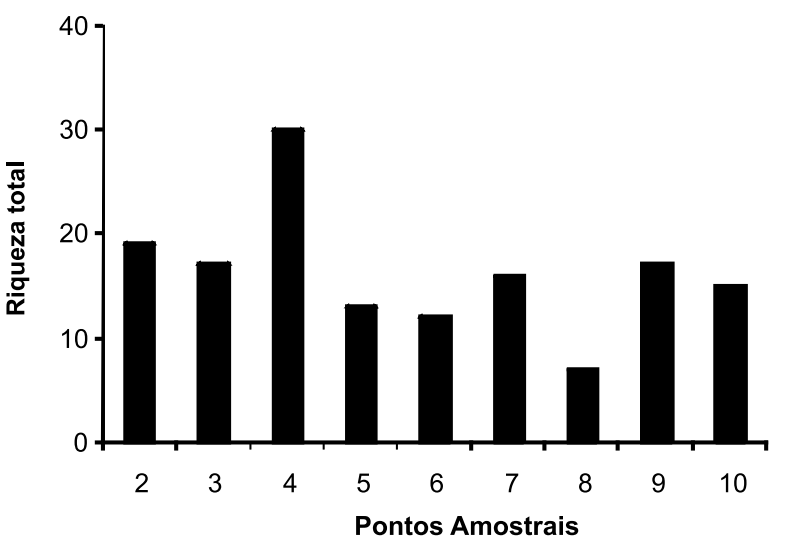

Figura 35 - Riqueza total do fitoplâncton para os 9 pontos amostrais na Bacia Hidrográfica do Rio Camboriú para o verão de 2005.

\section{Euglenophyceae}
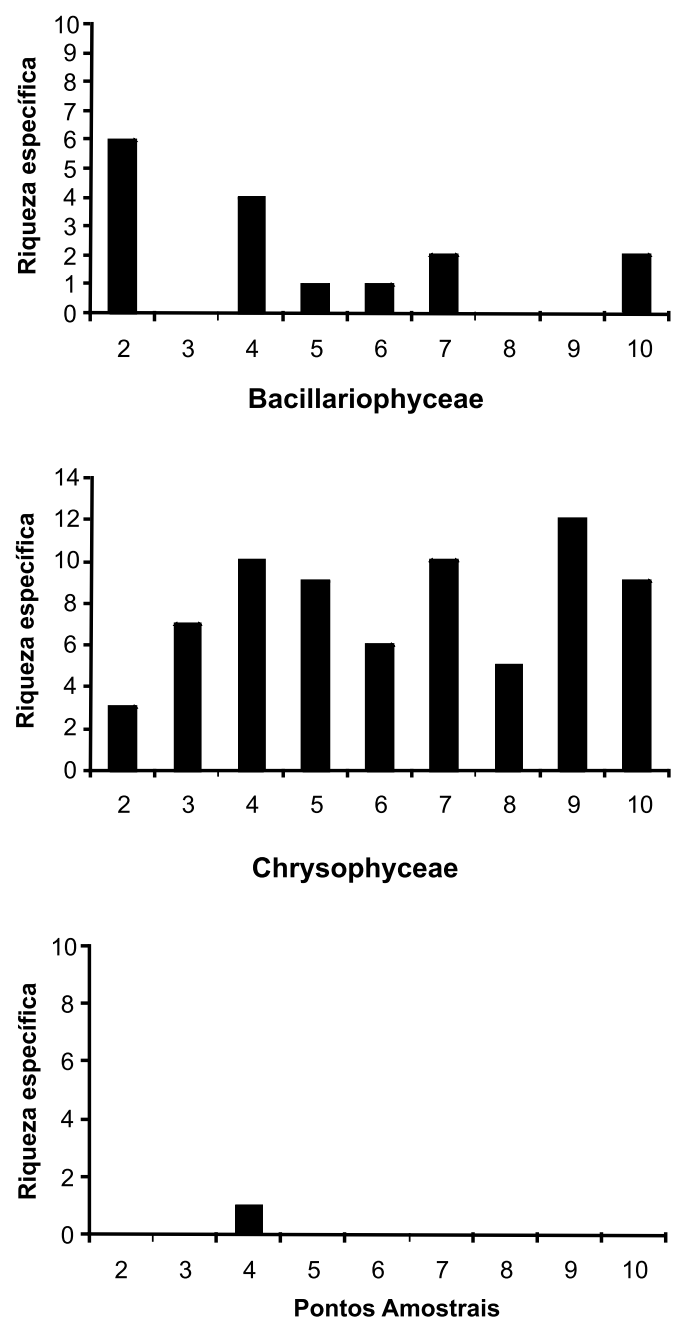

Figura 36 - Riqueza específica do fitoplâncton para os 9 pontos amostrais na Bacia Hidrográfica do Rio Camboriú referente às classes Zygnematophyceae, Euglenophyceae, Cyanophyceae, Chlorophyceae, Bacillariophyceae e Crysophyceae para o verão de 2005. 


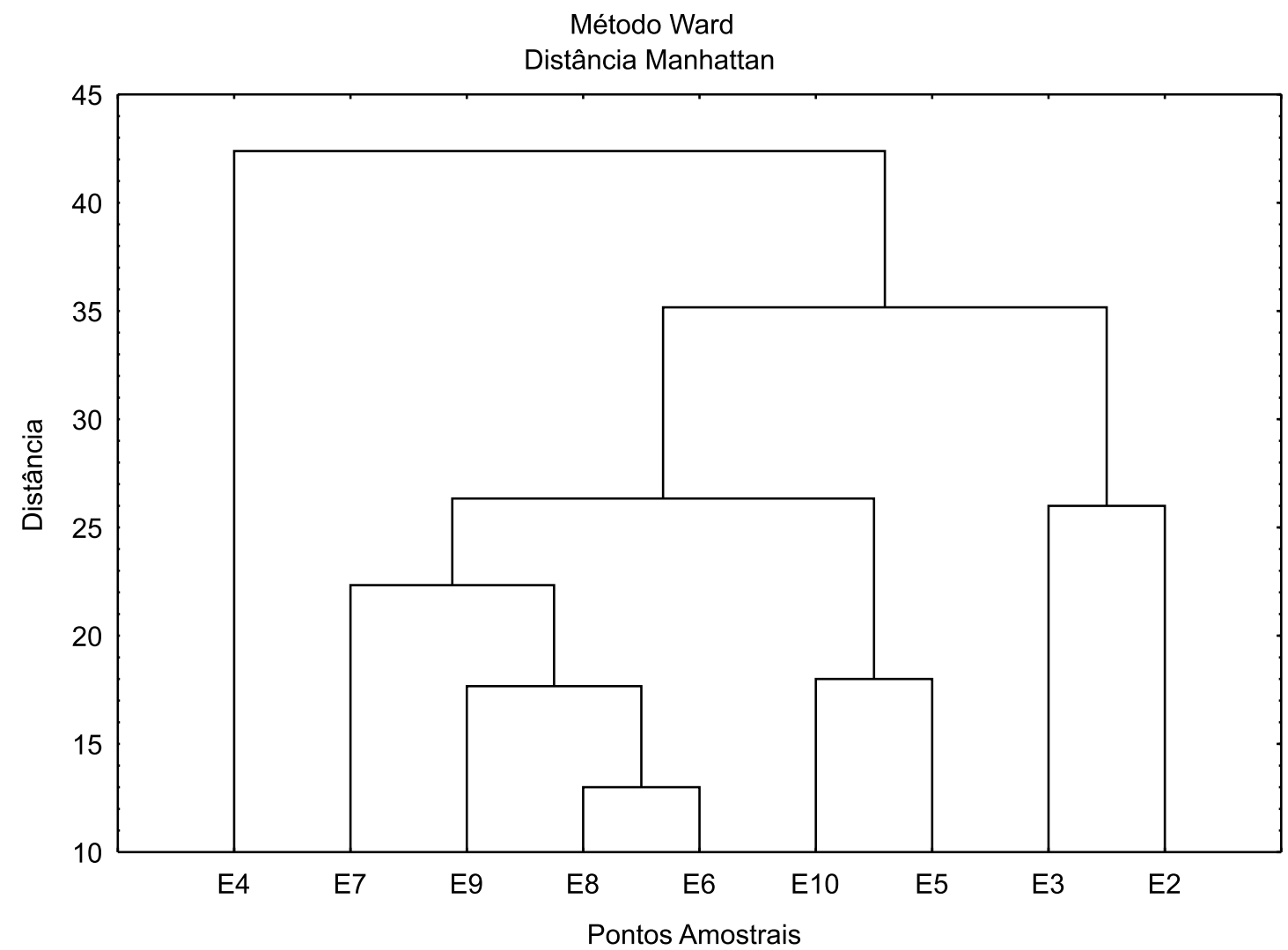

Figura 37 - Análise de similaridade (cluster) para a matriz de presença e ausência de 87 taxa do fitoplâncton identificados em 9 pontos amostrais na Bacia Hidrográfica do Rio Camboriú (SC) no verão de 2005.

vadas ambientalmente e com baixos valores de riqueza. O segundo grupo é constituído pelos pontos 5 e 10, os quais possuem valores de riqueza relativamente baixos e estão situados em áreas rurais, sendo afetados diretamente pela atividade de rizicultura e pecuária. O terceiro grupo abrange os pontos 2 e 3 , pontos localizados em áreas urbanas com maiores valores de riqueza específica. O ponto amostral 4 , que está situado em área peri-urbana, ficou isolado na análise de similaridade, fato que deve estar associado à maior riqueza de fitoplâncton encontrada neste local e por apresentar maior número de organismos com exclusividade de ocorrência (Synura sp.). Estes resultados salientam que além dos fatores naturais que influenciam a distribuição das assembléias fitoplanctônicas em ambientes lóticos, a atividade antropogênica também exerce influência na composição e distribuição destes organismos neste ecossistema aquático.

\section{CONCLUSÕES}

Este estudo mostra a alta riqueza de espécies do fitoplâncton encontradas em diferentes áreas da Bacia Hidrográfica do Rio Camboriú (SC) em uma cole- ta de verão. Os pontos amostrais localizados na região potamal apresentaram valores de riqueza elevados, possivelmente devido a baixa velocidade da corrente, 0 que conseqüentemente favorece a acumulação de nutrientes e o estabelecimento do fitoplâncton. Ao contrário, na região ritral a riqueza de espécies foi menor, devido ao aumento da velocidade da corrente, carreando nutrientes e interferindo no estabelecimento das assembléias algais. Além da composição do fitoplâncton na Bacia Hidrográfica do Rio Camboriú ser influenciada por características naturais (relação ritral-potamal), fatores associados à atividade antropogênica em diferentes áreas do ambiente (áreas urbanas, peri-urbanas, rurais e naturais) também determinam a composição de sua estrutura.

\section{AGRADECIMENTOS}

Agradecemos ao Programa Integrado de PósGradução e Gradução (PIPG) da UNIVALI pelo apoio através de bolsa de iniciação científica. 


\section{REFERÊNCIAS}

Bicudo, C.E.M. \& Menezes, M. 2006. Gêneros de algas de águas continentais do Brasil (chave para identificação e descrições). 2o Edição. RiMa, São Carlos, 489p.

Bourrelly, P. 1985. Les algues d'eau douce. Initiation à la systematique. Tome I: Les algues vertes. Societé Nouvelle des Éditions Boubéé, Paris, 572p.

Burliga, A.L.; Torgan, L.C.; Nobrega, E.A.; Beaumord, A.C.; Costa, C.O. \& Yamauti, D.V. 2005. Diatomáceas epilíticas do rio Itajaí-Mirim, Santa Catarina, Brasil. Acta Sci. Biol. Sci. 27(4):415-421.

Germain, H. 1981. Flore des Diatomées. Scocieté Nouvelle de Éditions Boubée, Paris, 441p.

Hegewald, E. 2000. New combination in the genus Desmodesmus

(Chlorophyceae, Scenedesmaceae). Algological Studies. 96:1-18.

Hoek, C.V.; Mann, D.G. \& Jahns, H.M. 1995. Algae, an introdution of phycology. Cambridge University Press, Cambridge, 627p.

Hürlimann, J.; Schanz, F. \& Uehlinger, U. 1991. Use of algae for monitoring rivers in switzerland. In: Whitton, B.A.; Rott, E. \& Friedrich, G. (ed.) Use of algae for monitoring rivers. Studia StudentenförderungsGes.m.b.H., Innsbruck. 169-173pp.

Krammer, R. 2000. Diatoms of Europe: Diatoms of the European Waters and Comparable Habitats. The genus Pinnularia sp. A.R.G. Gantner Verlag K.G., Meerbush, 703p.

Lange-Bertalot, H. 2001. Diatoms of Europe: Diatoms of the European Waters and Comparable Habitats. Navicula sensu stricto. 10 genera separated from Navicula sensu lacto Frustulia. A.R.G. Gantner Verlag K.G., Meerbush, 526p.

Lobo, E.A., Callegaro, V.L.M. \& Bender, E.P. 2002. Utilização de algas diatomáceas epilíticas como indicadoras da qualidade da água em rios e arroios da região hidrográfica do Guaíba, RS, Brasil. EDUNISC, Santa Cruz do Sul, 127p.
Miranda, A.L.B.; Beaumord, A.C. \& Mello, R.S.P. 2003. Aplicação do índice de especificidade de hábitat em Chlrococcales fitoplanctônicas, como indicador de qualidade ambiental em rios costeiros: estudo de caso do rio Itajaí-Mirim, SC, Brasil. Notas Técnicas da Facimar. 7:109-117.

Pereira-Filho, J.; Schettini, C. A. F.; Rörig, L. \& Siegle, E. 2001. Intratidal variation and net transport of dissolved inorganic nutrients, POC and chlorophyll a in the Camboriú river estuary, Brazil. Estuarine, Coastal and Shelf Science. 53:249-257.

Reviers, B. 2006. Biologia e filogenia das algas. Tradução lara Maria Franceschini. ARTMED, Porto Alegre, 280p.

Rosa, Z. M. \& Miranda-Kiesslich, A. L. 1988. O gênero Pediastrum Meyen (ChlorococcalesHydrodictyaceae) do Sistema Lagunar da região litoral do Rio Grande do Sul, Brasil. Iheringia, Ser. Bot. 38:149-169.

Sant'anna, C.L.; Azevedo, M.T.P.; Agujaro, L.F.; CarvaIho, M.C.; Carvalho, L.R. \& Souza, R.C.R. 2006. Manual ilustrado para identificação e contagem de cianobactérias planctônicas de águas continentais brasileiras. Interciência, Rio de Janeiro, 58p.

Tamanaha, M.S. 2004. Estrutura das assembléias fitoplanctônicas e testes de inibição de crescimento algal: avaliação dos efeitos dos herbicidas e inseticidas utilizados na cultura do arroz irrigado na região de Itajaí/SC. Tese de Mestrado. Universidade do Vale do Itajaí. 92p.

Tucci, A.; Deberdt, G.L.B. \& Deberdt, A.J. 2004. Análise da comunidade de fitoplâncton do reservatório de Salto Grande (Americana, SP): uma revisão dos estudos desenvolvidos em um sistema eutrófico. In: Espíndola, E.L.G.; Leite, M.A. \& Dornfeld, C.B. Reservatório de Salto Grande (Americana, SP): caracterização, impactos e proposta de manejo. RiMa, São Carlos. 107-153pp.

Urban, S. R. 2003. Análise da qualidade da água da bacia do rio Camboriú, SC. Monografia de Graduação. Universidade do Vale do Itajaí. 76p. 